\title{
Strong Fubini properties of ideals
}

\author{
by \\ Ireneusz R e c ław (Gdańsk) and Piotr Z a krzewski (Warszawa)
}

\begin{abstract}
Let $I$ and $J$ be $\sigma$-ideals on Polish spaces $X$ and $Y$, respectively. We say that the pair $\langle I, J\rangle$ has the Strong Fubini Property (SFP) if for every set $D \subseteq X \times Y$ with measurable sections, if all its sections $D_{x}=\{y:\langle x, y\rangle \in D\}$ are in $J$, then the sections $D^{y}=\{x:\langle x, y\rangle \in D\}$ are in $I$ for every $y$ outside a set from $J$ ("measurable" means being a member of the $\sigma$-algebra of Borel sets modulo sets from the respective $\sigma$-ideal). We study the question of which pairs of $\sigma$-ideals have the Strong Fubini Property. Since CH excludes this phenomenon completely, sufficient conditions for SFP are always independent of ZFC.

We show, in particular, that:

- if there exists a Lusin set of cardinality the continuum and every set of reals of cardinality the continuum contains a one-to-one Borel image of a non-meager set, then $\langle\operatorname{MGR}(X), J\rangle$ has SFP for every $J$ generated by a hereditary $\Pi_{1}^{1}$ (in the Effros Borel structure) family of closed subsets of $Y(\operatorname{MGR}(X)$ is the $\sigma$-ideal of all meager subsets of $X)$,

- if there exists a Sierpiński set of cardinality the continuum and every set of reals of cardinality the continuum contains a one-to-one Borel image of a set of positive outer Lebesgue measure, then $\left\langle\mathrm{NULL}_{\mu}, J\right\rangle$ has SFP if either $J=\mathrm{NULL}_{\nu}$ or $J$ is generated by any of the following families of closed subsets of $Y\left(\mathrm{NULL}_{\mu}\right.$ is the $\sigma$-ideal of all subsets of $X$ having outer measure zero with respect to a Borel $\sigma$-finite continuous measure $\mu$ on $X)$ :
\end{abstract}

(i) all compact sets,

(ii) all closed sets in $\mathrm{NULL}_{\nu}$ for a Borel $\sigma$-finite continuous measure $\nu$ on $Y$,

(iii) all closed subsets of a $\Pi_{1}^{1}$ set $A \subseteq Y$.

1. Preliminaries. This paper, though self-contained, may be viewed as a continuation of [16], where the question which pairs of $\sigma$-ideals have

1991 Mathematics Subject Classification: Primary 04A15, 28A05; Secondary 03E05.

Key words and phrases: Polish space, Strong Fubini Property, $\sigma$-ideal, cardinal coefficients, measurability.

Both authors were partially supported by the Alexander von Humboldt Foundation while they were visiting FU-Berlin and TU-Berlin, respectively; the first author was partially supported by BW/5100-5-0272-7, the second author was partially supported by KBN grant 2 P03A 04709. 
the Fubini Property was studied. Our notation and terminology is therefore consistent with [16] and in fact in most cases follows [14].

Recall that, motivated by the classical Fubini and Kuratowski-Ulam theorems (see [14], 8.41), we say that a pair $\langle I, J\rangle$ of $\sigma$-ideals on Polish spaces $X$ and $Y$, respectively, has the Fubini Property (FP) if for every Borel set $B \subseteq X \times Y$, if all its sections $B_{x}=\{y:\langle x, y\rangle \in B\}$ are in $J$, then the sections $B^{y}=\{x:\langle x, y\rangle \in B\}$ are in $I$ for every $y$ outside a set from $J$. By a $\sigma$-ideal on $X$ we mean here a proper subfamily of $\mathcal{P}(X)$ containing all singletons and closed under taking subsets and countable unions. We usually (but, unlike [16], not always) assume that for any $A \in I$ there is $B \in \mathbf{B}(X) \cap I$ with $A \subseteq B$, i.e., $I$ has a Borel basis.

Relaxing the condition that $B \in \mathbf{B}(X \times Y)$ we say that the pair $\langle I, J\rangle$ has the Strong Fubini Property (SFP) if for every $D \subseteq X \times Y$ with all sections $D^{y}$ measurable, i.e., $\forall y D^{y} \in \mathbf{B}_{I}$, where $\mathbf{B}_{I}$ is the $\sigma$-algebra of Borel sets modulo sets from $I$, if all sections $D_{x}$ are in $J$, then the sections $D^{y}$ are in $I$ for every $y$ outside a set from $J$. Note that in general we cannot omit the condition of measurability of horizontal sections of $D$, since we have the following (however, see [12] and the notes after 1.11 and 1.12)

Example 1.1. Suppose $I$ is a $\sigma$-ideal on $X$ and $\kappa=\operatorname{non}(I)$ (see below). Take $E \subseteq X$ with $|E|=\kappa$; let $\leq$ be a well-ordering of $E$ of order type $\kappa$ and set

$$
D=\{\langle x, y\rangle: y \leq x\} .
$$

Then $\forall x D_{x} \in I$ but $\left\{y: D^{y} \notin I\right\}=E \notin I$.

Also note that under $\mathrm{CH}$ no pair $\langle I, J\rangle$ has SFP. Indeed, let us say that a set $D \subseteq X \times Y$ is a 0-1 counterexample to SFP for $\langle I, J\rangle$ if $\forall x \in X D_{x} \in J$ and $\forall y \in Y D^{y} \in I^{*}$, where $I^{*}=\{X \backslash A: A \in I\}$. Then we have

Example 1.2. Assume $\mathrm{CH}$ and, given Polish spaces $X, Y$, let $D \subseteq X \times Y$ be such that $\forall x \in X\left|D_{x}\right| \leq \aleph_{0}$ and $\forall y \in Y\left|X \backslash D^{y}\right| \leq \aleph_{0}$. Then $D$ is a 0-1 counterexample to SFP for any pair $\langle I, J\rangle$ of $\sigma$-ideals on $X$ and $Y$, respectively.

Consequently, the sufficient conditions for SFP we are looking for in this paper are always independent of ZFC.

In the next couple of facts we reformulate the definitions and present some basic properties of the notions introduced above; we always assume that $I$ and $J$ are $\sigma$-ideals on Polish spaces $X$ and $Y$, respectively. The proofs are routine and we omit them.

Lemma 1.3. For a set $D \subseteq X \times Y$ the following are equivalent:

(i) $\forall x D_{x} \in J$,

(ii) $\forall E \notin J \bigcap_{y \in E} D^{y}=\emptyset$. 
Proposition 1.4 (cf. [12], Lemma 1). The following are equivalent:

(i) $\langle I, J\rangle$ has $S F P$,

(ii) if $Z \subseteq Y, Z \notin J$ and $\left\{D^{y}: y \in Z\right\} \subseteq \mathbf{B}_{I} \backslash I$, then there exists $E \subseteq Z$ with $E \notin J$ such that $\bigcap_{y \in E} D^{y} \neq \emptyset$.

Note that if $I$ has a Borel basis, then in 1.4(ii) as well as in the definition of the SFP above, we can assume that $\forall y D^{y} \in \mathbf{B}(X)$.

Proposition 1.5. The following are equivalent:

(i) there is no 0-1 counterexample to SFP for $\langle I, J\rangle$,

(ii) if $\left\{D^{y}: y \in Y\right\} \subseteq I^{*}$, then there exists $E \subseteq Y$ with $E \notin J$ such that $\bigcap_{y \in E} D^{y} \neq \emptyset$.

(iii) if $\left\{C_{x}: x \in X\right\} \subseteq J^{*}$, then there exists $E \subseteq X$ with $E \notin I$ such that $\bigcap_{x \in E} C_{x} \neq \emptyset$.

Note that SFP for $\langle I, J\rangle$ does not necessarily imply SFP for $\langle J, I\rangle$. On the other hand, $D$ is a $0-1$ counterexample to SFP for $\langle I, J\rangle$ iff $\{\langle y, x\rangle$ : $\langle x, y\rangle \in(X \times Y) \backslash D\}$ is a 0-1 counterexample to SFP for $\langle J, I\rangle$.

We say that $I$ and $J$ are isomorphic and write $I \equiv J$ if there exists a bijection $f: X \rightarrow Y$ between $X$ and $Y$ such that for $A \subseteq X, A \in I \Leftrightarrow$ $f[A] \in J$. If there exists such a Borel isomorphism $f$, then we say that $I$ and $J$ are Borel isomorphic, in symbols $I \equiv_{B} J$. It is well known that the $\sigma$-ideal MGR(X) of all meager subsets of $X$ is Borel isomorphic to the $\sigma$-ideal $\mathbb{K}=\operatorname{MGR}(\mathbb{R})$, assuming $X$ has no isolated points. It is also well known that every $\sigma$-ideal of the form $\mathrm{NULL}_{\mu}$ for a $\sigma$-finite continuous (i.e., vanishing on singletons) Borel measure $\mu$ on $X$ is Borel isomorphic to the $\sigma$-ideal $\mathbb{L}$ of Lebesgue measure zero sets.

Proposition 1.6 (cf. [16], 1.4). Suppose that $I^{\prime}$ and $J^{\prime}$ are $\sigma$-ideals on $X$ and $Y$, respectively.

(i) If $I \equiv_{B} I^{\prime}$ and $J \equiv J^{\prime}$, then $\langle I, J\rangle$ has $S F P$ if and only if $\left\langle I^{\prime}, J^{\prime}\right\rangle$ does.

(ii) If $I \equiv I^{\prime}$ and $J \equiv J^{\prime}$, then there is no 0-1 counterexample to SFP for $\langle I, J\rangle$ if and only if there does not exist one for $\left\langle I^{\prime}, J^{\prime}\right\rangle$.

Recall that given $A \notin I$, the restriction of $I$ to $A$, denoted by $I \mid A$, is the $\sigma$-ideal on $X$ given by

$$
I \mid A=\{C \subseteq X: C \cap A \in I\} .
$$

Note that if $I$ has a Borel basis and $A \in \mathbf{B}(X)$, then $I \mid A$ has a Borel basis as well.

Proposition 1.7 (cf. [16], 3.8). (i) Let $\bar{I}$ be a $\sigma$-ideal on $X$ with a Borel basis such that $I \subseteq \bar{I}$ and let $A \subseteq Y$ with $A \notin J$. Then $S F P$ for $\langle I, J\rangle$ implies SFP for $\langle\bar{I}, J \mid A\rangle$. 
(ii) If each $Z \subseteq Y$ with $Z \notin J$ contains $A \notin J$ such that the pair $\langle I, J \mid A\rangle$ has $S F P$, then so does the pair $\langle I, J\rangle$.

The following cardinal invariants of $\sigma$-ideals will turn out to be relevant to SFP:

- $\operatorname{non}(I)=\min \{\kappa: \exists B \notin I|B| \leq \kappa\}$,

- $\operatorname{non}_{l}(I)=\min \left\{\kappa: \forall A \in \mathbf{B}_{I} \backslash I \exists B \notin I B \subseteq A \wedge|B| \leq \kappa\right\}$,

- $\operatorname{shr}(I)=\min \{\kappa: \forall A \notin I \exists B \notin I B \subseteq A \wedge|B| \leq \kappa\}$,

- $\operatorname{cov}(I)=\min \{|\mathcal{F}|: \mathcal{F} \subseteq I \wedge \bigcup \mathcal{F}=\mathbb{R}\}$,

- $\operatorname{cov}_{l}(I)=\min \left\{|\mathcal{F}|: \mathcal{F} \subseteq I \wedge \bigcup \mathcal{F} \in \mathbf{B}_{I} \backslash I\right\}$,

- $\operatorname{add}(I)=\min \{|\mathcal{F}|: \mathcal{F} \subseteq I \wedge \bigcup \mathcal{F} \notin I\}$,

- $\operatorname{cof}(I)=\min \{|\mathcal{F}|: \mathcal{F} \subseteq I \wedge \forall A \in I \exists B \in \mathcal{F} A \subseteq B\}$.

The notation $\operatorname{shr}(I)$ is taken from Kada and Yuasa [11]. Note that:

$$
\begin{aligned}
\operatorname{non}_{l}(I) & =\min \left\{\operatorname{non}(I \mid A): A \in \mathbf{B}_{I} \backslash I\right\}, \\
\operatorname{shr}(I) & =\min \{\operatorname{non}(I \mid A): A \notin I\}, \\
\operatorname{cov}_{l}(I) & =\min \left\{\operatorname{cov}(I \mid A): A \in \mathbf{B}_{I} \backslash I\right\}, \\
\operatorname{add}(I) & =\min \{\operatorname{cov}(I \mid A): A \notin I\}, \\
\operatorname{non}(I) & \leq \operatorname{non}_{l}(I) \leq \operatorname{shr}(I) \leq \operatorname{cof}(I) \quad(\text { see }[11], 2.1) .
\end{aligned}
$$

It is also known that the inequality non $(I)<\operatorname{shr}(I)$ is consistent with ZFC for $I \in\{\mathbb{L}, \mathbb{K}, \mathcal{K}\}$, where $\mathcal{K}$ is the $\sigma$-ideal of $\sigma$-bounded subsets of the Baire space $\mathbb{N}^{\mathbb{N}}$ (see [11], 2.9).

We say that $I$ is $c c c$ if there is no uncountable family of disjoint sets in $\mathbf{B}_{I} \backslash I$. If $I$ is not ccc, then SFP for $\langle I, J\rangle$ is rather unlikely to hold.

Proposition 1.8. If there is a disjoint family of cardinality non $(J)$ consisting of sets from $\mathbf{B}_{I} \backslash I$, then the pair $\langle I, J\rangle$ does not have SFP.

The idea behind SFP, expressed in 1.4 above, that every family of " $J$ positively" many " $I$-positive" sets always has " $J$-positively" many members with nonempty intersection, has been around for some time. Here is a sample of results.

Proposition 1.9 (Fremlin, [7], 1Ec). Suppose that I is ccc. If $\operatorname{cov}_{l}(I)>$ $\omega_{1}$, then the pair $\langle I,[Y] \leq \omega\rangle$ has SFP.

NoTE. In the terminology of $[7]$ this amounts to saying that if the measurable space with negligibles $\left(X, \mathbf{B}_{I}, I\right)$ is $\omega_{2}$-Baire, then there is no uncountable point-countable family in $\mathbf{B}_{I} \backslash I$.

Proposition 1.10 (Silver, see [13]). Assume that $\lambda<\kappa \leq \boldsymbol{c}$ are regular uncountable cardinals such that there is a $\lambda$-saturated $\kappa$-additive ideal $I$ on $X$ (this requires $\kappa$ to be quasi-measurable - see [8], 9C). Then the pair $\langle I,[Y] \leq \lambda\rangle$ has SFP. 
Note. Silver's lemma is more general: If $\mathcal{I}$ is a $\lambda$-saturated $\kappa$-additive ideal on $\kappa$, where $\lambda<\kappa$ are regular uncountable cardinals, then given $\left\{C_{\alpha}\right.$ : $\alpha<\lambda\} \subseteq \mathcal{P}(\kappa) \backslash \mathcal{I}$, there is $E \subseteq \lambda$ with $|E|=\lambda$ so that $\bigcap_{\alpha \in E} C_{\alpha} \neq \emptyset$ (compare 1.4 above).

Proposition 1.11 (Kunen, see [12], Proposition 3). Assume that $\kappa \leq \boldsymbol{c}$ is a regular uncountable cardinal such that there is a $\kappa$-additive measure $m: \mathcal{P}(X) \rightarrow[0,1]$ and let $I=\{A \subseteq \kappa: m(A)=0\}$ (this requires $\kappa$ to be atomlessly-measurable - see [8]). Then the pair $\left\langle I, \mathrm{NULL}_{\nu}\right\rangle$ has SFP for any $\sigma$-finite continuous measure $\nu$ on $Y$.

Note. Kunen's theorem is more general: If $I$ is as above then, given $Z \subseteq Y$ with $Z \notin$ NULL $_{\nu}$ and $\left\{C^{y}: y \in Z\right\} \subseteq \mathcal{P}(X) \backslash I$, there exists $E \subseteq Z$ with $E \notin \mathrm{NULL}_{\nu}$ such that $\bigcap_{y \in E} C^{y} \neq \emptyset$.

Proposition 1.12 (Kamburelis, [12], Proposition 2). Assume that $\kappa \leq \boldsymbol{c}$ is a regular uncountable cardinal and there is a $\kappa$-additive ideal $I$ on $X$ such that the quotient Boolean algebra $\mathcal{P}(X) / I$ is isomorphic to $\mathbb{B}_{\lambda} / \mathbb{K}_{\lambda}$ for some $\lambda$, where $\mathbb{B}_{\lambda}$ is the $\sigma$-algebra generated by the basic open sets in $2^{\lambda}$ and $\mathbb{K}_{\lambda}$ is the $\sigma$-ideal of meager sets in $2^{\lambda}$. Then the pair $\langle I, \operatorname{MGR}(Y)\rangle$ has SFP.

Note. Kamburelis's theorem is more general in exactly the same sense as Kunen's theorem above.

There is a vast literature concerning strong Fubini theorems, i.e., statements about the existence and equality of iterated integrals of functions which are not necessarily measurable. This is, of course, implicitly related to SFP for $I=J=\mathbb{L}$ (see [9], [6], [18], [8] and [19]). In particular, it is shown in $[19]$ that SFP for $\langle\mathbb{L}, \mathbb{L}\rangle$ follows from the Measure Extension Axiom of Carlson (see [4]) stating that given any countable collection of subsets of $\mathbb{R}$, the Lebesgue measure can be extended to a countably additive measure which measures all of them. SFP for $\langle\mathbb{K}, \mathbb{K}\rangle$ follows from a similar category extension axiom (see [20], Theorem 3.1).

The second author learnt the strongest result in this direction from D. H. Fremlin, who mentioned that he himself had heard about it from H. Woodin, in the context of random real model. It amounts, modulo 2.4(ii), to the following

TheOREM 1.13 (folklore?). If $\operatorname{shr}(\mathbb{L})<\operatorname{cov}(\mathbb{L})$, then for every set $D \subseteq$ $\mathbb{R} \times \mathbb{R}$ with all sections Lebesgue measurable there exists a Borel set $B \subseteq \mathbb{R} \times \mathbb{R}$ such that $\mathbb{L}$-almost all respective sections of $D$ and $B$ are $\mathbb{L}$-almost equal.

It is known that a model for $\omega_{1}=\operatorname{shr}(\mathbb{L})<\operatorname{cov}(\mathbb{L})\left(\omega_{1}=\operatorname{shr}(\mathbb{K})<\right.$ $\operatorname{cov}(\mathbb{K})$, resp.) is the model obtained by adding $\kappa>\omega_{1}$ many random reals (Cohen reals, resp.) to a model of $\mathrm{CH}$ (see e.g. [11], 2.6, but it was already known to Kunen and Solovay in the early 70's). 
The main results of this paper present various conditions which imply SFP for $\langle I, J\rangle$.

In the first part we study the conditions having the form of inequalities between cardinal invariants of $I$ and $J$. In particular, we exploit the strength of the inequality $\operatorname{shr}(J)<\operatorname{cov}(I)$. This leads to generalizations of 1.13 to the cases where $I=\mathbb{K}$ or $\mathbb{L}$ and $J$ is an arbitrary ccc $\sigma$-ideal with a Borel basis (Theorems 2.14 and 2.16).

In the second part we look at conditions asserting the existence of special sets related to $I$. In particular, we show that for $I=\mathbb{K}$ (L , resp.) if there exists a Lusin (Sierpiński, resp.) set of cardinality the continuum and every set of reals of cardinality the continuum contains a one-to-one Borel image of a nonmeager (not Lebesgue measurable, resp.) set, then $\langle I, J\rangle$ has SFP for every "reasonable" $\sigma$-ideal $J$ provided that $\langle I, J\rangle$ has FP (Theorem 3.1).

In the final part we collect some necessary conditions for SFP and indicate open problems.

Throughout the paper we assume that $I$ and $J$ are $\sigma$-ideals on Polish spaces $X$ and $Y$, respectively.

2. Sufficient conditions - cardinal invariants. We say that $L \subseteq X$ is an $I$-Lusin set if $L$ is uncountable and for every $A \in I, A \cap L$ is countable.

The following is an easy generalization of Fremlin's theorem 1.9.

Proposition 2.1. Suppose that $I$ is ccc. If $\operatorname{cov}_{l}(I)>\omega_{1}$ and for each $Z \notin J$ there is a J-Lusin set $L \subset Z$, then the pair $\langle I, J\rangle$ has SFP.

Proof. This follows from 1.7 and 1.9. It is enough to notice that if $L$ is an arbitrary $J$-Lusin set, then $J\left|L=[Y] \leq \aleph_{0}\right| L$, so $\langle I, J \mid L\rangle$ has SFP.

Corollary 2.2. Suppose that I is ccc. If $\operatorname{cov}_{l}(I)>\omega_{1}$ and $\operatorname{cof}(J)=\omega_{1}$, then the pair $\langle I, J\rangle$ has SFP.

Proof. This follows from 2.1 since $\operatorname{cof}(J)=\omega_{1}$ implies that each $Z \notin J$ contains a $J$-Lusin set.

Corollary 2.3. Suppose that $I$ is ccc and $J$ is generated by all Borel subsets of a $\Pi_{1}^{1}$ non-Borel set $A \subseteq Y$. If $\operatorname{cov}_{l}(I)>\omega_{1}$, then the pair $\langle I, J\rangle$ has SFP.

Proof. This follows from 2.2, because each Borel subset of $A$ is covered by countably many constituents of $A$; consequently, $\operatorname{cof}(J)=\omega_{1}$.

If $A, B \subseteq X$ we say that $A, B$ are equal modulo $I$, in symbols $A={ }_{I} B$, if the symmetric difference $A \triangle B \in I$. If $\phi(x)$ is a formula and $\{x \in X$ : $\phi(x)\}={ }_{I} X$, then we say that $\phi(x)$ holds for $I$-almost all $x \in X$.

Suppose that $I$ is ccc. If $A \subseteq X$ then by $A^{*}$ we denote the unique, modulo $I$, set from $\mathbf{B}_{I}$ such that $A \subseteq A^{*_{I}}$ and $\forall B \in \mathbf{B}_{I} B \subseteq A^{*_{I}} \backslash A \Rightarrow$ 
$B \in I$. To see that this definition is correct, consider a maximal family of pairwise disjoint sets in $\mathbf{B}_{I} \backslash I$ disjoint from $A$. If, additionally, $I$ has a Borel basis, then we always assume that $A^{*_{I}} \in \mathbf{B}$. In any case, if $A \in \mathbf{B}_{I}$, then $A^{* I}={ }_{I} A$.

The following lemma reveals the key property of the cardinal invariants $\operatorname{non}_{l}(I)$ and $\operatorname{shr}(I)$ in the case $I$ is ccc.

Lemma 2.4. Suppose that I is ccc.

(i) For every set $A \in \mathbf{B}_{I} \backslash I$ there exists a set $E \subseteq A$ such that $|E| \leq$ $\operatorname{non}_{l}(I)$ and $E^{*}{ }_{I} A$. In particular, there exists a set $Z \subset X$ such that $|Z| \leq \operatorname{non}_{l}(I)$ and $Z^{*_{I}}=X$.

(ii) For every set $A \subseteq X$ with $A \notin I$, there exists a set $E \subseteq A$ such that $|E| \leq \operatorname{shr}(I)$ and $A^{*_{I}}=E^{*^{I}}$.

Proof. This is proved by an exhaustion argument. Let $\kappa=\operatorname{non}_{l}(I)$ $(\kappa=\operatorname{shr}(I)$, resp.). In each case it suffices to find $E \subseteq A$ with $|E| \leq \kappa$ and $A \backslash E^{* I} \in I$. So suppose that there is no such $E$. To reach a contradiction, construct inductively a sequence of subsets of $A$ with the following properties:

- $\forall \alpha\left|C_{\alpha}\right| \leq \kappa$,

- $\forall \alpha C_{\alpha} \notin I$,

- $\forall \alpha, \beta\left(\alpha \neq \beta \Rightarrow C_{\alpha}^{* I} \cap C_{\beta}^{* I}=\emptyset\right)$.

At step $\beta<\omega_{1}$ let $E_{\alpha}=\bigcup_{\alpha<\beta} C_{\alpha}$ and using the fact that $A \backslash E_{\alpha}^{*_{I}} \notin I$ find $C_{\alpha} \subseteq A \backslash E_{\alpha}^{*_{I}}$ with $\left|C_{\alpha}\right| \leq \kappa$ and $C_{\alpha} \notin I$.

The sequence $\left\langle C_{\alpha}^{* I}: \alpha<\omega_{1}\right\rangle$ contradicts the fact that $I$ is ccc.

Proposition 2.5. Suppose that $I$ is ccc. If $\operatorname{non}_{l}(I)<\operatorname{add}(J)$, then the pair $\langle I, J\rangle$ has SFP.

Proof. Let $\kappa=\operatorname{non}_{l}(I)$. By $2.4(\mathrm{i})$ there is $Z \subset X$ such that $|Z| \leq \kappa$ and $Z^{*}=X$.

Take $D \subset X \times Y$ with $\forall x D_{x} \in J$ and $\forall y D^{y} \in \mathbf{B}_{I}$. Let $W=\bigcup_{x \in Z} D_{x}$. Then $W \in J$, since $\kappa<\operatorname{add}(J)$ and we have $\left\{y: D^{y} \notin I\right\} \subseteq W$. Indeed,

$$
\forall y \in Y \backslash W D^{y} \cap Z=\emptyset,
$$

so $D^{y} \subset X \backslash Z$ and consequently, since $D^{y} \in \mathbf{B}_{I}, D^{y} \in I$.

As a corollary we obtain a generalization of 3.10(iii) from [16].

Proposition 2.6. Assume Martin's Axiom + there is a quasi-measurable cardinal $\kappa<\boldsymbol{c}$ (see [7], 9G). Let $A \subseteq X$ be a set of cardinality $\kappa$ and let $\mathcal{I}$ be an $\omega_{1}$-saturated $\kappa$-additive ideal on $A$. If $\operatorname{add}(J)=\boldsymbol{c}$ and $I$ is the $\sigma$-ideal on $X$ with basis consisting of Borel sets $B \subseteq X$ such that $B \cap A \in \mathcal{I}$, then the pair $\langle I, J\rangle$ has SFP. 
Proof. Clearly, $I$ is ccc and $\operatorname{non}_{l}(I)=\kappa<\operatorname{add}(J)$.

We now turn to the consequences of the inequality $\operatorname{shr}(J)<\operatorname{cov}(I)$ which apparently is the most important cardinal condition for SFP. Part (i) of our next lemma belongs to the folklore.

Lemma 2.7. (i) If either $\operatorname{non}(J)<\operatorname{cov}(I)$ or $\operatorname{non}(I)<\operatorname{cov}(J)$, then there is no 0-1 counterexample to SFP for $\langle I, J\rangle$.

(ii) If either $\operatorname{shr}(J)<\operatorname{cov}(I)$ or $\operatorname{non}(I)<\operatorname{add}(J)$, then for every $Z \subseteq Y$ with $Z \notin J$, there is no 0-1 counterexample to SFP for $\langle I, J \mid Z\rangle$.

Proof. (i) follows immediately from 1.5. (ii) is a direct application of (i) to the pair $\langle I, J \mid Z\rangle$ where $Z \notin J$.

Given a semigroup $G$ of Borel functions from $X$ to $X$ we say that $I$ is:

- $G$-invariant if $g^{-1}[A] \in I$ for any $g \in G$ and $A \in I$;

- $G$-ergodic if $\bigcup_{g \in G} g^{-1}[A] \in I^{*}$ for any $A \in \mathbf{B}_{I} \backslash I$.

Note that the $\sigma$-ideals $\mathbb{K}$ and $\mathbb{L}$ are invariant and ergodic under the group of rational translations.

LEMma 2.8. Suppose that there exists a countable semigroup $G$ of Borel functions from $X$ to $X$ such that the $\sigma$-ideal $I$ is $G$-invariant and $G$-ergodic. Then the following are equivalent:

(i) $\langle I, J\rangle$ has $S F P$;

(ii) for every $Z \subseteq Y$, if $Z \notin J$, then there is no 0-1 counterexample to $S F P$ for $\langle I, J \mid Z\rangle$.

Proof. Clearly, (i) $\Rightarrow$ (ii). To see that (ii) $\Rightarrow$ (i), take $D \subseteq X \times Y$ with $\forall x D_{x} \in J$ and $\forall y D^{y} \in \mathbf{B}_{I}$. Suppose that $Z=\left\{y: D^{y} \notin I\right\} \notin J$. Then $E=\bigcup_{z \in Z}\left(\left(\bigcup_{g \in G} g^{-1}\left[D^{z}\right]\right) \times\{z\}\right)$ is a 0-1 counterexample to SFP for $\langle I, J \mid Z\rangle$.

Lemmas 2.7(ii) and 2.8 immediately give

TheOREM 2.9. Suppose that there exists a countable semigroup $G$ of Borel functions such that the $\sigma$-ideal $I$ is $G$-invariant and $G$-ergodic. If either $\operatorname{shr}(J)<\operatorname{cov}(I)$ or $\operatorname{non}(I)<\operatorname{add}(J)$, then the pair $\langle I, J\rangle$ has SFP. In particular, if $I \in\{\mathbb{K}, \mathbb{L}\}$, then $\operatorname{shr}(J)<\operatorname{cov}(I)$ implies SFP for $\langle I, J\rangle$.

In the forthcoming paper [21] the second author proves that $\mathrm{FP}$ for $\langle\mathbb{K}, J\rangle$ $\left(\langle\mathbb{L}, J\rangle\right.$, resp.) implies that in the model obtained by adding $\kappa>\omega_{1}$ many Cohen reals (random reals, resp.) to a model of $\mathrm{CH}$ we have $\operatorname{shr}(J)<\operatorname{cov}(\mathbb{K})$ $(\operatorname{shr}(J)<\operatorname{cov}(\mathbb{L})$, resp.), provided that $J$ has sufficiently good absoluteness properties. In particular, the latter is the case when either $J$ is a Suslin $\sigma$-ideal (see [1], 3.7) or $J$ is generated by a hereditary $\boldsymbol{\Pi}_{1}^{1}$ (in the Effros Borel structure - see [14], 35.G) family of closed subsets of $Y$. A different 
approach to the consistency of SFP for $\langle I, J\rangle$ when $I \in\{\mathbb{K}, \mathbb{L}\}$ will be presented in Section 3.

The sufficient condition for SFP given in the next theorem is apparently of a different character. However, in some important cases it will turn out to be situated between $\operatorname{shr}(J)<\operatorname{cov}(I)$ and SFP for $\langle I, J\rangle$ (see 2.13 below).

First we need a folklore-like lemma.

Lemma 2.10. Suppose that $J$ has a Borel basis and $E \subseteq Y$. If $\mathcal{P}(E)$ $\subseteq \mathbf{B}_{J}$, then $E \in J$.

Pro of. Let $E \in \mathbf{B}_{J} \backslash J$; we will prove that $\mathcal{P}(E) \nsubseteq \mathbf{B}_{J}$.

Shrinking $E$ if necessary, we can assume that $E \in \mathbf{B} \backslash J$. Then $E$ is an uncountable Borel subset of a Polish space, so it contains a set $B$ which is neither disjoint from nor contains an uncountable Borel subset of $E$ (a Bernstein set). By a classical argument, $B \notin \mathbf{B}_{J}$.

Theorem 2.11. Suppose that $J$ has a Borel basis. If for every $D \subseteq X \times Y$, the conditions $\forall x D_{x} \in \mathbf{B}_{J}$ and $\forall y D^{y} \in \mathbf{B}_{I}$ imply that $\left\{y: D^{y} \in I\right\} \in \mathbf{B}_{J}$, then the pair $\langle I, J\rangle$ has SFP.

Proof. Take an arbitrary $D \subseteq X \times Y$ such that $\forall x D_{x} \in J$ and $\forall y D^{y} \in \mathbf{B}_{I}$. Let $E=\left\{y: D^{y} \notin I\right\}$. Note that $\mathcal{P}(E) \subseteq \mathbf{B}_{J}$. Indeed, if $C \subseteq E$, then $C=\left\{y:(\widetilde{D})^{y} \notin I\right\}$ where $\widetilde{D}=D \cap(X \times C)$ has all sections measurable. Consequently, $C \in \mathbf{B}_{J}$ by the hypotheses of the theorem. Hence 2.10 implies that $E \in J$.

Lemma 2.12. Suppose that $J$ is ccc and let $\kappa=\operatorname{shr}(J)$. For any disjoint sets $A_{1}, A_{2} \subseteq Y$ which cannot be separated by a set from $\mathbf{B}_{J}$ there are sets $E_{1} \subseteq A_{1}$ and $E_{2} \subseteq A_{2}$ with $\left|E_{1}\right|,\left|E_{2}\right| \leq \kappa$ which cannot be separated by a set from $\mathbf{B}_{J}$.

Proof. Clearly, $A_{1}, A_{2} \notin J$ since otherwise they could be separated by a set from $J$. Now it suffices to apply 2.4(ii) and let $E_{1} \subseteq A_{1}$ and $E_{2} \subseteq A_{2}$ be such that $\left|E_{1}\right|,\left|E_{2}\right| \leq \kappa$ and $A_{i}^{* J}=E_{i}^{* J}$ for $i=0,1$.

In view of 2.11 the following is a strengthening of 2.9 in the case $J$ is ccc and has a Borel basis.

TheOREM 2.13. Suppose that $J$ is ccc with a Borel basis and there exists a countable semigroup $G$ of Borel functions from $X$ to $X$ such that $I$ is $G$-invariant and $G$-ergodic. If $\operatorname{shr}(J)<\operatorname{cov}(I)$, then for every $D \subseteq X \times Y$, the conditions $\forall x D_{x} \in \mathbf{B}_{J}$ and $\forall y D^{y} \in \mathbf{B}_{I}$ imply that $\left\{y: D^{y} \in I\right\} \in \mathbf{B}_{J}$. In particular, this implication is true for $I \in\{\mathbb{K}, \mathbb{L}\}$.

Proof. Take $D \subseteq X \times Y$ with $\forall x D_{x} \in \mathbf{B}_{J}$ and $\forall y D^{y} \in \mathbf{B}_{I}$. Let $A_{1}=\left\{y: D^{y} \in I\right\}$ and $A_{2}=Y \backslash A_{1}$. We want to prove that $A_{1} \in \mathbf{B}_{J}$. 
Set $\widetilde{D}=\bigcup_{y \in Y}\left(\left(\bigcup_{g \in G} g^{-1}\left[D^{y}\right]\right) \times\{y\}\right)$. Note that since $I$ is $G$-invariant, $G$-ergodic and $|G| \leq \aleph_{0}$ we have

$$
\forall y \in A_{1}(\widetilde{D})^{y} \in I \text { and } \forall y \in A_{2}(\widetilde{D})^{y} \in I^{*} .
$$

By 2.12 , there are sets $E_{1} \subseteq A_{1}$ and $E_{2} \subseteq A_{2}$ with $\left|E_{1}\right|,\left|E_{2}\right| \leq \operatorname{shr}(J)$ which cannot be separated by a set from $\mathbf{B}_{J}$.

Set $C=\bigcup_{y \in E_{1}}(\widetilde{D})^{y} \cup \bigcup_{y \in E_{2}}\left(X \backslash(\widetilde{D})^{y}\right)$. Since $\left|E_{1} \cup E_{2}\right|<\operatorname{cov}(I), C \neq X$; pick $x \in X \backslash C$. Then $E_{1} \cap D_{x}=\emptyset$ and $E_{2} \subseteq D_{x}$, so $D_{x}$ separates $E_{1}$ from $E_{2}$. But $D_{x} \in \mathbf{B}_{J}$ and we have reached a contradiction.

We get further strengthenings when we restrict to the cases $I=\operatorname{MGR}(X)$ or $\mathrm{NULL}_{\mu}$ for a Borel $\sigma$-finite continuous measure $\mu$ on $X$. Let $\mathrm{BP}=$ $\mathbf{B}_{\mathrm{MGR}(X)}$ and $\operatorname{MEAS}_{\mu}=\mathbf{B}_{N U L L_{\mu}}$. We also write $={ }^{*}$ instead of $=\operatorname{MGR}(X)$.

TheOREM 2.14. Suppose that $J$ is ccc and has a Borel basis. If $\operatorname{shr}(J)<$ $\operatorname{cov}(\mathbb{K})$, then for every $D \subseteq X \times Y$, the conditions $\forall x D_{x} \in \mathbf{B}_{J}$ and $\forall y D^{y} \in$ $B P$ imply that there exists a set $B$ which is Borel in $X \times Y$ and such that for $J$-almost all $y \in Y$ the horizontal sections $D^{y}$ and $B^{y}$ are equal modulo meager sets.

Proof. Let $\left\{U_{n}: n \in \mathbb{N}\right\}$ be an open basis for $Y$. Define

$$
B^{\prime}=\bigcup_{n}\left(U_{n} \times\left\{y: D^{y} \text { is comeager in } U_{n}\right\}\right) .
$$

Then 2.13 easily implies that for each $n,\left\{y: D^{y}\right.$ is comeager in $\left.U_{n}\right\} \in \mathbf{B}_{J}$; let $P_{n} \in \mathbf{B}(Y)$ be such that $\left\{y: D^{y}\right.$ is comeager in $\left.U_{n}\right\}={ }_{J} P_{n}$. Set

$$
B=\bigcup_{n}\left(U_{n} \times P_{n}\right) \text {. }
$$

Then $B \in \mathbf{B}(X \times Y)$ and for $J$-almost all $y$ we have

$$
B^{y}=^{*}\left(B^{\prime}\right)^{y}=\bigcup\left\{U_{n}: D^{y} \text { is comeager in } U_{n}\right\}={ }^{*} D^{y}
$$

(see [14], 8.29).

As a corollary we get a strong approximation theorem for sets with all sections having $\mathrm{BP}$.

TheOREM 2.15. If $\operatorname{shr}(\mathbb{K})<\operatorname{cov}(\mathbb{K})$, then for every set $D \subseteq X \times Y$ with all sections $D_{x}$ and $D^{y}$ having $B P$ there exists a Borel set $B \subseteq X \times Y$ such that almost all respective sections of $D$ and $B$ are almost equal; here "almost" means modulo meager sets in the respective spaces.

Proof. By 2.14, there is a Borel set $B \subseteq X \times Y$ with $B^{y}={ }^{*} D^{y}$ for almost all $y$. Let $E=D \triangle B$. Then $E^{y} \in \operatorname{MGR}(X)$ for almost all $y$. But by $2.9, \operatorname{since} \operatorname{shr}(\mathbb{K})<\operatorname{cov}(\mathbb{K})$, we have $\operatorname{SFP}$ for $\langle\operatorname{MGR}(Y), \operatorname{MGR}(X)\rangle$. Consequently, $E_{x} \in \operatorname{MGR}(Y)$ for almost all $x$. Thus $B_{x}={ }^{*} D_{x}$ for almost all $x$. 
If $\mathcal{S}$ is a $\sigma$-algebra on a set $Z$ and $E \subseteq Z$, then $\mathcal{S} \mid E=\{A \cap E: A \in \mathcal{S}\}$ is the relative $\sigma$-algebra on $Z$.

The following result is a generalization of 1.13. The proof of (i) below is just a refinement of the argument (modulo 2.4(ii)) the second author learnt from D. H. Fremlin. We present it here for the sake of completeness thanks to the kind permission of David Fremlin. A considerable simplification of the original proof of (ii), which resulted in dropping the unnecessary assumption $\operatorname{non}(\mathbb{L})<\operatorname{cov}(\mathbb{L})($ see $[17])$, was also suggested by him.

TheOREM 2.16. Suppose that $J$ is ccc with a Borel basis and $\mu$ is a $\sigma$-finite Borel continuous measure on $X$. If $\operatorname{shr}(J)<\operatorname{cov}(\mathbb{L})$, then for every $D \subseteq X \times Y$ with $\forall x D_{x} \in \mathbf{B}_{J}$ and $\forall y D^{y} \in \operatorname{MEAS}_{\mu}$ :

(i) the function $y \mapsto \mu\left(D^{y}\right)$ is $\mathbf{B}_{J}$-measurable,

(ii) there exists a set $B$ which is Borel in $X \times Y$ and such that for J-almost all $y \in Y$ the horizontal sections $D^{y}$ and $B^{y}$ are equal modulo $\mu$-null sets.

Proof. Since $\operatorname{NULL}_{\mu} \equiv_{B} \mathbb{L}$, we can assume that $X=[0,1]$ and $\mu$ is the Lebesgue measure on $[0,1]$. Let $\mathcal{A}$ be the $\sigma$-algebra of subsets of $[0,1] \times Y$ with all sections measurable, i.e.,

$$
\mathcal{A}=\left\{A \subseteq[0,1] \times Y: \forall x A_{x} \in \mathbf{B}_{J} \wedge \forall y A^{y} \in \mathrm{MEAS}_{\mu}\right\} .
$$

Let $D \in \mathcal{A}$.

(i) Define $g(y)=\mu\left(D^{y}\right)$ for $y \in Y$. We want to prove that $g$ is $\mathbf{B}_{J^{-}}$ measurable.

Claim 1. For every $E \subseteq Y$, if $|E|<\operatorname{cov}(\mathbb{L})$, then the function $g \mid E$ is $\mathbf{B}_{J} \mid$ E-measurable.

Proof. Write $\mu_{\mathbb{N}}$ for the natural product measure on $[0,1]^{\mathbb{N}}$. The Strong Law of Large Numbers (see [8], A2X) tells us that for all $y \in Y$ and for $\mu_{\mathbb{N}}$-almost all $z=\left\langle z_{i}: i \in \mathbb{N}\right\rangle \in[0,1]^{\mathbb{N}}$,

$$
g(y)=\lim _{n \rightarrow \infty} \frac{1}{n}\left|\left\{i<n: z_{i} \in D^{y}\right\}\right| .
$$

Since $|E|<\operatorname{cov}(\mathbb{L})=\operatorname{cov}\left(\mathrm{NULL}_{\mu_{\mathbb{N}}}\right)$, it follows that there is $z \in[0,1]^{\mathbb{N}}$ such that $(*)$ holds for all $y \in E$.

Let

$$
h(y)=\lim _{n \rightarrow \infty} \frac{1}{n}\left|\left\{i<n: z_{i} \in D^{y}\right\}\right| \quad \text { for } y \in Y .
$$

We have $g|E=h| E$, so it is now enough to prove that $h$ is $\mathbf{B}_{J}$-measurable. For this purpose fix $n \in \mathbb{N}$ and let

$$
h_{n}(y)=\left|\left\{i<n: z_{i} \in D^{y}\right\}\right| \quad \text { for } y \in Y .
$$


We have $h_{n}(y)=\left|\left\{i<n: y \in D_{z_{i}}\right\}\right|$, so for any $k \in \mathbb{N}$,

$$
\left\{y: h_{n}(y)=k\right\}=\bigcup_{|T|=k}\left(\bigcap_{i \in T} D_{z_{i}} \cap \bigcap_{i \in n \backslash T}\left([0,1] \backslash D_{z_{i}}\right)\right) \in \mathbf{B}_{J} .
$$

Thus for each $n, h_{n}$ is $\mathbf{B}_{J}$-measurable and so is $h$.

Claim 2. For every function $f: Y \rightarrow \mathbb{R}$, if $f$ is not $\mathbf{B}_{J}$-measurable, then there exists a set $E \subseteq Y$ such that $|E| \leq \operatorname{shr}(J)$ and the function $f \mid E$ is not $\mathbf{B}_{J} \mid$ E-measurable.

Pr o o f. It clearly suffices to prove that for every $C \subseteq Y$, if $C \notin \mathbf{B}_{J}$, then there exists a set $E \subseteq Y$ such that $|E| \leq \operatorname{shr}(J)$ and $C \cap E \notin \mathbf{B}_{J} \mid E$.

So take an arbitrary $C \subseteq Y$ with $C \notin \mathbf{B}_{J}$ and note that $C$ and $Y \backslash C$ cannot be separated by a set from $\mathbf{B}_{J}$. Hence, by 2.12 , there are $E_{1} \subseteq C$ and $E_{2} \subseteq Y \backslash C$ with $\left|E_{1}\right|,\left|E_{2}\right| \leq \operatorname{shr}(J)$ which cannot be separated by a set from $\mathbf{B}_{J}$. Set $E=E_{1} \cup E_{2}$. It is easy to see that this works.

Finally, Claims 1 and 2 together with the condition $\operatorname{shr}(J)<\operatorname{cov}(\mathbb{L})$ show immediately that $g$ is $\mathbf{B}_{J}$-measurable.

(ii) Consider the $\sigma$-ideal

$$
\mathcal{I}=\left\{C \subseteq[0,1] \times Y: C^{y} \in \mathbb{L} \text { for } J \text {-almost all } y \in Y\right\} .
$$

We want to prove that $D \in \mathbf{B}_{\mathcal{I}}$.

Define

$$
B^{\prime}=\left\{\langle x, y\rangle: x \text { is a point of density } 1 \text { of } D^{y}\right\} .
$$

By the Lebesgue Density Theorem (see [14], 17.9), $\forall y \mu\left(\left(B^{\prime}\right)^{y} \triangle D^{y}\right)=0$, thus $B^{\prime} \triangle D \in \mathcal{I}$ and it suffices to prove that $B^{\prime} \in \mathbf{B}_{\mathcal{I}}$.

Note that

$$
\langle x, y\rangle \in B^{\prime} \Leftrightarrow \lim _{n \rightarrow \infty} \frac{n}{2} \mu\left(D^{y} \cap\left[x-\frac{1}{n}, x+\frac{1}{n}\right]\right)=1,
$$

so it is enough to show that for any fixed $h>0$ the function $g$ given by

$$
g(x, y)=\mu\left(D^{y} \cap[x-h, x+h]\right) \quad \text { for } x \in \mathbb{R}, y \in Y
$$

is $\mathbf{B}_{\mathcal{I}}$-measurable.

To see the latter, first note that $\mathbf{B}_{\mathcal{I}}$ contains $\mathbf{B}_{J} \otimes \mathbf{B}(Y)$, the $\sigma$-algebra generated by the family $\left\{A \times B: A \in \mathbf{B}_{J}, B \in \mathbf{B}(Y)\right\}$. But

$$
g(x, y)=\mu\left((D \cap([x-h, x+h] \times Y))^{y}\right),
$$

so, by (i), the function $g$ is $\mathbf{B}_{J}$-measurable in the second variable. Since $g$ is also continuous in the first variable, it follows that it is $\mathbf{B}_{J} \otimes \mathbf{B}(Y)$ measurable.

For the sake of completeness we present, as a corollary, a strong approximation theorem for functions with all sections measurable. Another argument may be reconstructed with the help of 2.4(ii) from the proof of 
Proposition $6 \mathrm{~Kb}$ in [8]; a more general approximation theorem is obtained there as a consequence of the existence of an atomlessly measurable cardinal.

Theorem 2.17. Let $\mu$ and $\nu$ be $\sigma$-finite Borel continuous measures on Polish spaces $X$ and $Y$, respectively. If $\operatorname{shr}(\mathbb{L})<\operatorname{cov}(\mathbb{L})$, then:

(i) for every set $D \subseteq X \times Y$ with all sections $D_{x}$ and $D^{y}$ measurable there exists a Borel set $B \subseteq X \times Y$ such that almost all respective sections of $D$ and $B$ are almost equal,

(ii) for every function $f: X \times Y \rightarrow \mathbb{R}$ such that all its sections

$$
f_{x}: y \mapsto f(x, y): Y \rightarrow \mathbb{R}, \quad f^{y}: x \mapsto f(x, y): X \rightarrow \mathbb{R}
$$

are measurable, there exists a Borel function $g: X \times Y \rightarrow \mathbb{R}$ such that almost all respective sections of $f$ and $g$ are almost equal. Here "almost" means modulo null sets in the respective spaces.

Proof. To prove (i) argue exactly as in the proof of 2.15 using 2.16 instead of 2.13 .

(ii) follows from (i) with the help of the ordinary machinery of simple functions.

Recall that, by the remarks following 2.9, the model for 2.14 and 2.15 ( 2.16 and 2.17 , resp.) is the Cohen real model (random real model, resp.), provided that $J$ has sufficiently good absoluteness properties and the pair $\langle\mathbb{K}, J\rangle(\langle\mathbb{L}, J\rangle$, resp.) has FP. It remains, however, an open problem to find such a ccc $\sigma$-ideal $J$ with a Borel basis and with $J \not_{B} \mathbb{K}\left(J \not_{B} \mathbb{L}\right.$, resp. $)$ (see [16] and [21]).

3. Sufficient conditions-special sets of reals. The results of this section say roughly that for such ideals $I$ as $\mathbb{K}$ and $\mathbb{L}, \mathrm{FP}$ and SFP for $\langle I, J\rangle$ are consistently the same, provided $J$ is "reasonable".

Given $\sigma$-algebras $\mathcal{S}_{1}$ and $\mathcal{S}_{2}$ on sets $Z_{1}$ and $Z_{2}$, respectively, we say that a map $f: Z_{1} \rightarrow Z_{2}$ is $\mathcal{S}_{1}-\mathcal{S}_{2}$-measurable if $f^{-1}[A] \in \mathcal{S}_{1}$ for any $A \in \mathcal{S}_{2}$.

Recall that $J$ is $\boldsymbol{\Sigma}_{\alpha}^{0}$-supported if for any $A \in I$ there is $B \in \boldsymbol{\Sigma}_{\alpha}^{0} \cap J$ with $A \subseteq B$.

Theorem 3.1. Suppose that:

(i) I has a Borel basis and is $c c c$,

(ii) the Boolean algebra $\mathbf{B}_{I} / I$ is homogeneous,

(iii) there exists an I-Lusin set of cardinality the continuum,

(iv) every subset of $X$ of cardinality the continuum contains a one-toone Borel image of a set not in $I$,

(v) there is $\alpha<\omega_{1}$ such that $J$ is $\boldsymbol{\Sigma}_{\alpha}^{0}$-supported,

(vi) for every Borel set $B \subseteq X \times Y$ we have $\left\{x: B_{x} \in J\right\} \in \mathbf{B}_{I}$.

Then, if the pair $\langle I, J\rangle$ has FP, then it also has SFP. 
Proof. The proof is based on the following result which is interesting in its own right.

Lemma 3.2. If a $\sigma$-ideal $I$ on a Polish space $X$ satisfies conditions (i)-(iv) above, then for every countably generated $\sigma$-algebra $\mathcal{A}$ of subsets of $X$ there exist a set $Z \subseteq X$ with $Z \notin I$ and $a \mathbf{B}(Z)$-A-measurable function $\varphi: Z \rightarrow X$ such that:

1. $\forall A \in I \varphi^{-1}[A] \in I$,

2. $\forall A \in\left(\mathbf{B}_{I} \backslash I\right) \varphi^{-1}[A] \notin I$.

Let us assume this temporarily and now complete the proof of the theorem.

Take an arbitrary $D \subseteq X \times Y$ such that $\forall x D_{x} \in J$ and $\forall y D^{y} \in \mathbf{B}_{I}$.

Since $J$ is $\boldsymbol{\Sigma}_{\alpha}^{0}$-supported, we can cover $D$ by a set $C$ with $\forall x C_{x} \in \boldsymbol{\Sigma}_{\alpha}^{0} \cap J$. By a result of Bing, Bledsoe and Mauldin [2], there is a countably generated $\sigma$-algebra $\mathcal{A}$ of subsets of $X$ such that $C \in \mathcal{A} \otimes \mathbf{B}(Y)$, the $\sigma$-algebra generated by the family $\{A \times B: A \in \mathcal{A}, B \in \mathbf{B}(Y)\}$.

Let $Z \notin I$ and $\varphi: Z \rightarrow X$ be the objects whose existence is guaranteed by 3.2. Consider the set $E \subseteq Z \times Y$ defined by

$$
\langle x, y\rangle \in E \Leftrightarrow\langle\varphi(x), y\rangle \in C .
$$

Since $\varphi$ is $\mathbf{B}(Z)$ - $\mathcal{A}$-measurable and $C \in \mathcal{A} \otimes \mathbf{B}(Y)$, we have $E \in \mathbf{B}(Z \times Y)$. So fix a $B \in \mathbf{B}(X \times Y)$ with $E=B \cap(Z \times Y)$. Note that $\forall x E_{x}=C_{\varphi(x)} \in J$ so, by (vi) above, we can assume that $B_{x} \in J$ for $I$-almost all $x$.

Now, by FP for $\langle I, J\rangle$ applied to $B$, we have $\left\{y: B^{y} \notin I\right\} \in J$. But for each $y, B^{y} \supseteq E^{y}=\varphi^{-1}\left[C^{y}\right] \supseteq \varphi^{-1}\left[D^{y}\right]$ and, by $3.2, D^{y} \notin I \Rightarrow \varphi^{-1}\left[D^{y}\right] \notin I$. Thus

$$
\left\{y: D^{y} \notin I\right\} \subseteq\left\{y: B^{y} \notin I\right\} \in J
$$

and consequently, $\left\{y: D^{y} \notin I\right\} \in J$.

Finally, we prove 3.2 .

Proof of 3.2. Enlarging $\mathcal{A}$ if necessary, we can assume that $\mathbf{B}(X) \subseteq \mathcal{A}$. Moreover, since all uncountable Polish spaces are Borel isomorphic, we can assume that $X=2^{\mathbb{N}}$.

Let $L$ be an $I$-Lusin set of cardinality $\boldsymbol{c}$. Since $I$ has properties (i)-(iii), a proposition from [3] (p. 220) tells us that we can additionally assume that $|B \cap L|=\boldsymbol{c}$ for each $B \in \mathbf{B}_{I} \backslash I$.

Let $\left\{A_{n}: n \in \mathbb{N}\right\}$ be a set of generators of $\mathcal{A}$; we can clearly assume that it separates points in $2^{\mathbb{N}}$, i.e. for any distinct points $x, y \in 2^{\mathbb{N}}$ there is $A_{n}$ containing one of them but not the other.

For each $n$ set $C_{n}=A_{n} \cap L$. Let $f: L \rightarrow 2^{\mathbb{N}}$ be the Marczewski function of the sequence $\left\langle C_{n}: n \in \mathbb{N}\right\rangle$, i.e.

$$
f(x)(n)=1 \Leftrightarrow x \in C_{n} \quad \text { for } x \in L .
$$


Set $W=f[L]$. Since $f$ is one-to-one, we may define $g: W \rightarrow 2^{\mathbb{N}}$ by $g=f^{-1}$. Clearly, $g$ is $\mathbf{B}(W)$ - $\mathcal{A}$-measurable and $\forall A \in I\left|g^{-1}[A]\right|=|f[A \cap L]|=$ $|A \cap L| \leq \aleph_{0}$.

Let us consider a maximal disjoint collection $\mathcal{C}$ of sets $P \in \mathbf{B}\left(2^{\mathbb{N}}\right) \backslash I$ with associated Borel one-to-one functions $h_{P}: T_{P} \rightarrow W$ such that $T_{P} \notin I$ and if we let $\psi_{P}=g \circ h_{P}: T_{P} \rightarrow 2^{\mathbb{N}}$, then

$$
\forall A \subseteq P\left(A \in \mathbf{B}(P) \backslash I \Rightarrow \psi_{P}^{-1}[A] \notin I\right) .
$$

Clearly, $|\mathcal{C}| \leq \aleph_{0}$ and we claim that

$$
2^{\mathbb{N}} \backslash \bigcup \mathcal{C} \in I
$$

For suppose otherwise, i.e., $R=2^{\mathbb{N}} \backslash \bigcup \mathcal{C} \notin I$. Then $|f[R \cap L]|=|R \cap L|=$ $\boldsymbol{c}$, so by (iv) above, there is a set $T \subseteq 2^{\mathbb{N}}$ with $T \notin I$ and a Borel one-to-one function $h: T \rightarrow f[R \cap L]$. Let $\psi=g \circ h: T \rightarrow 2^{\mathbb{N}}$. Consider the $\sigma$-ideal $\bar{I}$ on $2^{\mathbb{N}}$ defined by

$$
\bar{I}=\left\{A \subseteq 2^{\mathbb{N}}: \exists B \in \mathbf{B}(R)\left(A \subseteq B \wedge \psi^{-1}[B \cap R] \in I\right)\right\} .
$$

Note that $I \subseteq \bar{I}$, since $\forall A \in I\left|\psi^{-1}[A]\right| \leq \aleph_{0}$, and $\bar{I}$ is proper, since $\psi^{-1}[R]=$ $T \notin I$. Since $I$ is ccc, there is a set $P \in \mathbf{B}\left(2^{\mathbb{N}}\right) \backslash I$ such that $\bar{I}=I \mid P$ (see [16], a remark preceding 3.4). Since $P \backslash R \in \bar{I} \mid P \subseteq I$, we can assume that $P \subseteq R$. It follows that

$$
\forall A \subseteq P\left(A \in \mathbf{B}(P) \backslash I \Rightarrow \psi^{-1}[A] \notin I\right) .
$$

But then $\langle P, h, T\rangle$ violates the maximality of $\mathcal{C}$, which proves $(*)$.

So let $\mathcal{C}=\left\{P_{n}: n \in \mathbb{N}\right\}$. We can further assume that the collection $\left\{T_{P_{n}}: n \in \mathbb{N}\right\}$ is disjoint. Indeed, it is not difficult to find a disjoint family $\left\{B_{n}: n \in \mathbb{N}\right\} \subseteq \mathbf{B}\left(2^{\mathbb{N}}\right) \backslash I$. But, since the Boolean algebra $\mathbf{B}_{I} / I$ is homogeneous, Sikorski's theorem (see [14], 15.10) tells us that for each $n$ there is a Borel isomorphism $\phi_{n}: B_{n} \rightarrow 2^{\mathbb{N}}$ between $B_{n}$ and $2^{\mathbb{N}}$ such that

$$
\forall A \in \mathbf{B}\left(2^{\mathbb{N}}\right) A \in I \Leftrightarrow \phi_{n}^{-1}[A] \in I .
$$

Since $I$ has a Borel basis, we can strengthen this to

$$
\forall A \subseteq 2^{\mathbb{N}} A \in I \Leftrightarrow \phi_{n}^{-1}[A] \in I .
$$

Now we can simply replace $T_{P_{n}}$ by $\phi_{n}^{-1}\left[T_{P_{n}}\right]$ and $h_{P_{n}}$ by $h_{P_{n}} \circ\left(\phi_{n} \mid \phi_{n}^{-1}\left[T_{P_{n}}\right]\right)$.

Let $Z=\bigcup_{n \in \mathbb{N}} T_{P_{n}}$ and define $\varphi: Z \rightarrow 2^{\mathbb{N}}$ by

$$
\varphi=\bigcup_{n \in \mathbb{N}} \psi_{P_{n}}
$$

We have $\forall A \in I\left|\varphi^{-1}[A]\right|=\aleph_{0}$, which proves part 1 .

To prove part 2 take an arbitrary $A \in \mathbf{B}_{I} \backslash I$. By part 1 , we can assume that $A \in \mathbf{B}\left(2^{\mathbb{N}}\right)$. By $(*)$ above, there is $n$ such that $A \cap P_{n} \notin I$. It follows 
that

$$
\varphi^{-1}[A] \supseteq\left(\psi_{P_{n}}\right)^{-1}\left[A \cap P_{n}\right] \notin I .
$$

Finally, the function $\varphi$ is $\mathbf{B}(Z)$ - $\mathcal{A}$-measurable as the union of countably many $\mathbf{B}(Z)-\mathcal{A}$-measurable functions.

We will now specialize the above taking into account the main results on FP established in [16].

TheOREM 3.3. Suppose that there exists a Lusin set of cardinality the continuum and every set of reals of cardinality the continuum contains a one-to-one Borel image of a nonmeager set. Suppose that $I$ is ccc and $\boldsymbol{\Sigma}_{2}^{0}$ supported and $J$ is generated by a hereditary $\boldsymbol{\Pi}_{1}^{1}$ (in the Effros Borel structure) family of closed subsets of $Y$. Then the pair $\langle I, J\rangle$ has SFP.

P r o of. By Theorem 2.4 of [16], the pair $\langle I, J\rangle$ has FP. Now we apply 3.1. Just recall that $I \equiv_{B} \operatorname{MGR}(X)$ (see [16], 1.3), so assumption (ii) is satisfied. Also, for every Borel set $B \subseteq X \times Y$ we have (see [14], 35.38)

$$
\left\{x: B_{x} \in J\right\} \in \mathbf{\Pi}_{1}^{1},
$$

which shows that (vi) holds as well.

Miller [15] proved that a model for the above is the Cohen real model (comp. the remarks following 2.9).

TheOREM 3.4. Suppose that there exists a Sierpinski set of cardinality the continuum and every set of reals of cardinality the continuum contains a one-to-one Borel image of a set of positive outer Lebesgue measure. Let $\mu$ be a $\sigma$-finite Borel continuous measure on $X$. Then the pair $\left\langle\mathrm{NULL}_{\mu}, J\right\rangle$ has SFP whenever $J$ is generated by any of the following families of closed subsets of $Y$ :

(i) all compact sets (in this case $Y$ is assumed to be non- $\sigma$-compact),

(ii) all closed sets in $\mathrm{NULL}_{\nu}$ for a $\sigma$-finite Borel continuous measure $\nu$ on $Y$,

(iii) all closed subsets of a $\Pi_{1}^{1}$ set $A \subseteq Y$.

Moreover, if $\nu$ is a $\sigma$-finite Borel continuous measure on $Y$, then the pair $\left\langle\mathrm{NULL}_{\mu}, \mathrm{NULL}_{\nu}\right\rangle$ has $S F P$.

Proof. By Theorem 2.5 of [16], the pair $\langle I, J\rangle$ has FP. Since in each of the cases (i)-(iii), $J$ is generated by a hereditary $\Pi_{1}^{1}$ family of closed subsets of $Y$, the possibility of applying 3.1 can be justified in exactly the same way as above.

The "moreover" part follows from 3.1 and the ordinary Fubini theorem.

Miller [15] proved that a model for the above is the random real model (comp. the remarks following 2.9). 
4. Necessary conditions and concluding remarks. There is a large gap between sufficient and necessary conditions for SFP. As a matter of fact we have nothing to say about the latter that would go beyond obvious generalizations of the necessary conditions given by Shipman [18] for the nonexistence of a 0-1 counterexample to SFP for $\langle\mathbb{L}, \mathbb{L}\rangle$ (a "generalized Sierpiński example" in Shipman's terminology). We state the following facts for the sake of completeness; the proofs are left to the reader (compare with [18]).

Proposition 4.1. (i) If there is no 0-1 counterexample to SFP for $\langle I, J\rangle$, then

$$
\min (\operatorname{non}(J), \operatorname{non}(I))<\boldsymbol{c} .
$$

(ii) If the pair $\langle I, J\rangle$ satisfies $S F P$, then $\min (\operatorname{shr}(J), \operatorname{non}(I))<\boldsymbol{c}$.

Proposition 4.2. If there is no 0-1 counterexample to SFP for $\langle I, I\rangle$, then:

(i) $X$ is not the union of any strictly increasing sequence $\left\langle A_{\alpha}: \alpha<\kappa\right\rangle$ of sets from $I$,

(ii) $\operatorname{non}(I)<\boldsymbol{c}$ and $\operatorname{add}(I)<\operatorname{cov}(I)$.

Finally, let us indicate some open questions.

(1) Does non $(I)<\operatorname{cov}(I)$ imply SFP for $\langle I, I\rangle$ at least for $I \in\{\mathbb{K}, \mathbb{L}\}$ ? Shipman claims that for $I=\mathbb{L}$ the answer is "yes" (see [18], p. 480) but he has never published a proof. Recall that by 2.9 , if $I \in\{\mathbb{K}, \mathbb{L}\}$ and $J$ is arbitrary, then SFP for $\langle I, J\rangle$ is implied by $\operatorname{shr}(J)<\operatorname{cov}(I)$.

(2) Does the nonexistence of a 0-1 counterexample to SFP for $\langle I, I\rangle, I \in$ $\{\mathbb{K}, \mathbb{L}\}$, imply SFP for $\langle I, I\rangle$ ? This is even stronger than (1). The restriction on $I$ is necessary: if $I=\mathbb{K} \cap \mathbb{L}$, then there is no 0 - 1 counterexample to SFP for $\langle I, I\rangle$ but FP for $\langle I, I\rangle$ does not hold (see [16], 3.9).

(3) Can we weaken the hypotheses of 3.1? In particular, does the existence of an $I$-Lusin set of cardinality the continuum, $I \in\{\mathbb{K}, \mathbb{L}\}$, plus the negation of CH imply SFP for $\langle I, I\rangle$ ? This in turn is weaker than (1), since it implies non $(I)<\operatorname{cov}(I)$.

(4) Is the simultaneous satisfaction of SFP for both $\langle\mathbb{K}, \mathbb{K}\rangle$ and $\langle\mathbb{L}, \mathbb{L}\rangle$ consistent with ZFC? Note that by 4.2 , this would imply $\boldsymbol{c}>\omega_{2}$ (see [6], where a similar problem is stated).

\section{References}

[1] T. Bartoszyński and H. Judah, Set Theory. On the Structure of the Real Line, A K Peters, 1995.

[2] R. H. Bing, W. W. Bledsoe and R. D. Mauldin, Sets generated by rectangles, Pacific J. Math. 51 (1974), 27-36. 
[3] J. Brzuchowski, J. Cichoń and B. Węglorz, Some applications of strong Lusin sets, Compositio Math. 43 (1981), 217-224.

[4] T. Carlson, Extending Lebesgue measure by infinitely many sets, Pacific J. Math. 115 (1984), 33-45.

[5] K. Eda, M. Kada and Y. Yuasa, The tightness about sequential fans and combinatorial properties, J. Math. Soc. Japan 49 (1997), 181-187.

[6] C. Freiling, Axioms of symmetry: throwing the darts at the real line, J. Symbolic Logic 51 (1986), 190-220.

[7] D. H. Fremlin, Measure-additive coverings and measurable selectors, Dissertationes Math. 260 (1987).

[8] - Real-valued-measurable cardinals, in: Set Theory of the Reals, H. Judah (ed.), Israel Math. Conf. Proc. 6 (1993), 151-304.

[9] H. Friedman, A consistent Fubini-Tonelli theorem for nonmeasurable functions, Illinois J. Math. 24 (1980), 390-395.

[10] P. R. Halmos, Measure Theory, Van Nostrand, 1950.

[11] M. Kad a and Y. Yuasa, Cardinal invariants about shrinkability of unbounded sets, Topology Appl. 74 (1996), 215-223.

[12] A. Kamburelis, A new proof of the Gitik-Shelah theorem, Israel J. Math. 72 (1990), 373-380.

[13] A. Kanamori and M. Magidor, The evolution of large cardinal axioms in set theory, in: Higher Set Theory, Lecture Notes in Math. 669, Springer, 1978, 99-275.

[14] A. S. Kechris, Classical Descriptive Set Theory, Grad. Texts in Math. 156, Springer, 1995.

[15] A. W. Miller, Mapping a set of reals onto the reals, J. Symbolic Logic 48 (1983), $575-584$.

[16] I. Recław and P. Zakrzewski, Fubini properties of ideals, submitted for publication.

[17] - - - Strong Fubini properties of ideals, preprint P 97-10, Institute of Math., Warsaw University.

[18] J. Shipman, Cardinal conditions for strong Fubini theorems, Trans. Amer. Math. Soc. 321 (1990), 465-481.

[19] P. Zakrzewski, Strong Fubini axioms from measure extension axioms, Comment. Math. Univ. Carolin. 33 (1992), 291-297.

[20] - Extending Baire Property by countably many sets, submitted for publication.

[21] —, Fubini properties of ideals and forcing, to appear.

Institute of Mathematics

University of Gdańsk

Wita Stwosza 57

80-952 Gdańsk, Poland

E-mail: matir@halina.univ.gda.pl
Institute of Mathematics University of Warsaw Banacha 2

02-097 Warszawa, Poland E-email: piotrzak@mimuw.edu.pl 